\title{
Conceitos e aplicabilidade da terapia fotodinâmica na periodontia: revisão de
}

\section{literatura}

\author{
Concepts and applicability of photodynamic therapy in periodontics: literature review \\ Conceptos y aplicabilidad de la fotodinámica en periodoncia: revisión de la literatura
}

Recebido: 07/11/2021 | Revisado: 13/11/2021 | Aceito: 16/11/2021 | Publicado: 26/11/2021

Juliane Kárin Pereira Pimentel

ORCID: https://orcid.org/0000-0002-8652-6075

Faculdade Independente do Nordeste, Brasil E-mail: julianekarin@hotmail.com

Karina Sarno Paes Alves Dias

ORCID: https://orcid.org/0000-0003-4840-2335

Faculdade Independente do Nordeste, Brasil

E-mail: karinasarnopad@gmail.com

\begin{abstract}
Resumo
O Introdução: A Terapia Fotodinâmica é uma reação fotoquímica dependente de oxigênio iniciada por laser de baixa potência com comprimento de onda apropriado. E tem sido amplamente utilizada na periodontia por permitir a redução de periodontopatógenos com ausência de efeitos sistêmicos colaterais e mínimas possibilidades de resistência bacteriana. Objetivo: Realizar uma revisão de literatura sobre a terapia fotodinâmica e a sua importância no tratamento de doenças do periodonto. Metodologia: Foi realizada uma pesquisa bibliográfica nas bases de dados Pubmed, Scielo e Lilacs, com o auxílio dos seguintes descritores em saúde: "Terapia Fotodinâmica" (Photodynamic Therapy); "Fotoquimioterapia" (Photochemotherapy); "Terapia a Laser de Baixa Potência" (Low Powe Laser Therapy) compreendidos entre os anos de 1991 e 2021. Resultados: Atentando para os fatos, viu-se que a escolha de um de um fotossensibilizador e a aplicação com um comprimento de onda correto e específico leva ao sucesso desta terapia. Conclusão: É necessário a realização de mais estudos voltados para a sua utilização no tratamento de doenças periodontais. Sendo definidos protocolos terapêuticos específicos que permitam sua execução baseada na ciência.
\end{abstract}

Palavras-chave: Terapia fotodinâmica; Fotoquimioterapia; Terapia com luz de baixa intensidade.

\begin{abstract}
Introduction: Photodynamic Therapy is an oxygen-dependent photochemical reaction initiated by a low-power laser with an appropriate wavelength. And it has been widely used in periodontics because it allows the reduction of periodontopathogens with no systemic side effects and minimal possibilities of bacterial resistance. Objective: To carry out a literature review on photodynamic therapy and its importance in the treatment of periodontal diseases. Methodology: A literature search was carried out in the Pubmed, Scielo and Lilacs databases, with the help of the following health descriptors: "Photodynamic Therapy" (Photodynamic Therapy); "Photochemotherapy" (Photochemotherapy); "Low Power Laser Therapy" between 1991 and 2021. Results: Looking at the facts, it was seen that choosing one of a photosensitizers and applying it with a correct and specific wavelength leads to the success of this therapy. Conclusion: It is necessary to carry out more studies aimed at its use in the treatment of periodontal diseases. Specific therapeutic protocols are defined that allow its execution based on science.
\end{abstract}

Keywords: Photodynamic therapy; Photochemotherapy; Low intensity light therapy.

\section{Resumen}

Introducción: La Terapia Fotodinámica es una reacción fotoquímica dependiente de oxígeno iniciada por un láser de baja potencia con una longitud de onda adecuada. Y ha sido muy utilizado en periodoncia porque permite la reducción de periodontopatógenos sin efectos secundarios sistémicos y con mínimas posibilidades de resistencia bacteriana. Objetivo: Realizar una revisión de la literatura sobre la terapia fotodinámica y su importancia en el tratamiento de las enfermedades periodontales. Metodología: Se realizó una búsqueda bibliográfica en las bases de datos Pubmed, Scielo y Lilacs, con la ayuda de los siguientes descriptores de salud: “Terapia Fotodinámica” (Terapia Fotodinámica); "Fotoquimioterapia" (fotoquimioterapia); "Terapia con láser de baja potencia" entre 1991 y 2021. Resultados: Mirando los hechos, se vio que elegir uno de los fotosensibilizadores y aplicarlo con una longitud de onda correcta y específica conduce al éxito de esta terapia. Conclusión: Es necesario realizar más estudios dirigidos a su uso en el tratamiento de las enfermedades periodontales. Se están definiendo protocolos terapéuticos específicos que permitan su ejecución basada en la ciencia.

Palabras clave: Terapia fotodinámica; Fotoquimioterapia; Terapia de luz de baja intensidad. 


\section{Introdução}

A periodontite é uma doença inflamatória de etiologia multifatorial e destrutiva progressiva dos tecidos de suporte, resultante de interações entre produtos bacterianos (Park et al., 2019). Dos patógenos mais importantes que causam doença periodontal, microrganismos complexos, como Aggregatibacter Actinomycetemcomitans, Porphyromonas gengivalis e Treponema denticola, Tannerella forsythia podem ser observadas (Tanner, 2014).

Devido ao fato de que o objetivo principal do tratamento periodontal é reduzir os agentes microbianos, os procedimentos de desbridamento mecânico (o uso de equipamentos ultrassônicos e manualmente), bem como o uso de antibióticos tópicos ou sistêmicos são considerados como as principais medidas terapêuticas no tratamento das doenças periodontais (Cobb, 2006).

Embora a utilização de antibióticos possa reduzir os patógenos periodontais, seu uso frequente pode causar resistência bacteriana, desencadear alergias, além da incapacidade de conseguir a concentração adequada da droga nas bolsas periodontais (Meisel \& Kocher 2005). Somando-se a isso, os antibióticos podem ocasionar efeitos colaterais como erupção cutânea, coceira, candidíase oral, náusea e vômito (Guillies et al., 2015). Também, devido à complexa anatomia da área da furca, a profundidade das bolsas e a penetração dos microrganismos nos tecidos, é difícil ter um acesso adequado para a realização da limpeza dessas regiões. Portanto, pelas razões descritas, os esforços para encontrar tratamento adjuvante aumentaram (Petersilka et al., 2003).

Um desses tratamentos é a terapia fotodinâmica (TFD) que se mostra bastante promissora. A TFD é uma reação fotoquímica dependente de oxigênio iniciada por laser de baixa potência com comprimento de onda apropriado, envolvendo o uso de um fármaco que é um corante fotoativo (fotossensibilizador) ativado pela luz de um comprimento de onda específico (Kurmar et al., 2015). Essa reação gera espécies reativas citotóxicas de oxigênio que têm meia-vida curta e efeito de raio limitado à área infectada, não afetando outros tecidos do hospedeiro (Moan \& Berg, 1991).

Dessa forma, devido à sua segurança e ausência de efeitos colaterais, a TFD constitui-se como um importante método terapêutico auxiliar para o desbridamento e alisamento radicular, a fim de aumentar a eficiência dos tratamentos periodontais não cirúrgicos. Tendo em vista os fatos mencionados, o objetivo do presente estudo foi apresentar, por meio de uma revisão de literatura, o conceito da TFD e avaliar os seus efeitos no tratamento das doenças periodontais.

\section{Metodologia}

Trata-se de uma revisão da literatura (Estrela, 2018) que foi realizada por meio de uma busca via online, utilizando as bases de dados Pubmed, Scielo e Lilacs, para o levantamento bibliográfico por meio dos seguintes descritores em saúde (DeCS): "Terapia Fotodinâmica" (Photodynamic Therapy); "Fotoquimioterapia" (Photochemotherapy); e "Terapia a Laser de Baixa Potência" (Low Power Laser Therapy).

A seleção dos artigos foi realizada de forma criteriosa e independente pelas autoras do presente estudo. Foi estabelecido como critério de inclusão na pesquisa, artigos em Inglês e Português abrangendo os anos de 1991 a 2021. Dissertações, trabalhos de conclusão de curso e artigos fora do período de tempo proposto foram excluídos do presente estudo.

\section{Revisão de Literatura}

As primeiras lesões periodontais são caracterizadas pela inflamação gengival, migração apical do epitélio juncional e exposição radicular. A destruição dos tecidos do ligamento periodontal, reabsorção óssea e mobilidade dental são consequências da periodontite, uma das maiores causas de perda dental nos adultos. A realização de técnicas manuais como a Raspagem e o Alisamento Radicular (RAR) proporcionam benefícios no tratamento periodontal através da redução do 
sangramento à sondagem, estabilização dos níveis de inserção clínica e diminuição da profundidade de sondagem clínica (Tsai et al., 2016).

Aspectos relacionados ao hospedeiro como anatomia complexa (região de furca, concavidades, sulcos e sítios distais de molares) e a capacidade de alguns microrganismos (Aggregatibacter actynomycetemcomitans, Porphyromonas gingivalis) invadirem os tecidos moles são fatores que limitam a eficácia do tratamento promovido pela RAR (Rajesh et al., 2011). Também o uso de antibióticos sistêmicos ou locais associados ao tratamento periodontal convencional é de grande valia, porém possui desvantagens devido a sua resistência, promovendo assim o desenvolvimento de infecções oportunistas, possuindo efeitos colaterais sistêmicos e discordância em relação à sua prescrição. Por apresentar essas desvantagens, foi necessário buscar mecanismos alternativos para controlar o biofilme e tratar as doenças periodontais (Zhao et al., 2021).

A terapia fotodinâmica integra-se na odontologia como uma técnica de fotodisinfecção (Prazmo et al., 2016) apresentando propriedades antibacterianas, parasitárias, virais e fúngicas. Tem sido amplamente utilizada devido a ausência de efeitos genotóxicos e mutagênicos, o que a torna um fator relevante a longo prazo (Konopka \& Goslinski, 2007). Esta reação ocorre pelo uso de oxigênio e radicais livres que são produzidos quando é ativada a luz desse agente fotosssensibilizador, se fazendo útil tanto no início da periodontite quanto na manutenção do tratamento (Javali et al., 2019). Através de um bom planejamento, o seu uso é bastante indicado para bolsas residuais, causando a inibição do efeito estimulador das citocinas próinflamatórias que são produzidas pelas células mononucleares, o que promove a diminuição da atividade biológica (Meimandi et al., 2017).

No entanto, os procedimentos de RAR e a TFD usados de forma separada apresentam evidências insuficientes para apoiar a sua eficácia (Sgolastra et al., 2011). Quando as técnicas são associadas ocorre uma significativa redução das bactérias e ação citotóxica sobre a Porphyromonas gingivalis, Prevotella intermedia, Tannerella forsythia, Treponema denticola e Aggregatibacter actinomycetemcomitans em pacientes com bolsa entre 5 e $6 \mathrm{~mm}$. A associação das técnicas tem sido amplamente aplicada em pacientes com diabetes mellitus tipo 2 e tabagistas, apresentando uma melhora no aspecto periodontal, imunológico e microbiológico (Al-hamoudi, 2017).

A TFD é considerada uma alternativa muito segura, não gera nenhum dano às células hospedeiras e foi aprovada pela Food and Drug Administration (FDA) (Garg et al., 2015). De acordo com Allison et al (2006), essa técnica envolve o uso de 3 componentes: Luz, Radicais livres de oxigênio e Fotossensibilizador.

A terapia envolve o uso do laser de baixa potência com comprimento de onda adequado para eliminar microrganismos tratados com drogas fotossensibilizadoras. O processo se baseia em um conjunto de reações foto-oxidativas, que ao desencadear alterações morfobiológicas, promovem necrose celular. Na periodontia, o agente fotossensibilizador é impregnado no biofilme subgengival, penetrando nas células bacterianas. A emissão da fonte de luz dos lasers ocasiona excitação do corante, desencadeando reações químicas que promovem geração de energia. O oxigênio das células ao absorver a energia liberada transforma-se em oxigênio singleto, que juntamente com os radicais livres altamente reativos, degradam polissacarídeos e destroem os sistemas biológicos bacterianos (Fernandes et al., 2009).

O fotossensibilizador tem função de localizar-se em um tipo de célula ou tecido específico, e sua ativação ocorre por meio da incidência da luz ocorrendo uma absorção (Carrera et al., 2016) (Figura 1). Quando o fotossensibilizador é estimulado por um comprimento de onda de luz apropriado (comprimentos de onda entre 650-900 nm que estão dentro da luz vermelha visível e próximo ao infravermelho), ele fornece radicais livres de oxigênio que causam danos aos tecidos. Os produtos citotóxicos têm meia-vida curta (cerca de $0,04 \mu \mathrm{s})$ e efeito de raio limitado $(0,20 \mu \mathrm{m})$ (Raghavendra et al., 2009).

Na prática odontológica os fotossensibilizadores mais preconizados são os corantes como o azul de metileno (MB) e o azul de toluidina (TBO) (Jiang et al., 2019). Com a intenção de extinguir as bactérias patogênicas visadas sem afetar os 
tecidos periodontais e evitar a morte de toda a flora bacteriana do local, impedindo que o hospedeiro fique suscetível a infecções oportunistas, tem sido utilizado fotossensibilizadores seletivos e hidrofóbicos (Habashneh et al., 2019).

Novas nanotecnologias foram desenvolvidas de forma rápida para direcionar a entrega de fotossensibilizadores hidrofóbicos em microrganismos para a melhoria do desempenho antimicrobiano (Qi et al., 2019). O ICG-nano com revestimento tem um grande potencial para ser um fotossensibilizador específico para a terapia fotodinâmica, pois é possível verificar o efeito do revestimento de quitosana sobre a capacidade de aderir ao periodonto e aos microrganismos (Nagahara $e t$ al., 2013).

Atentando para os fatos, viu-se que a escolha de um de um fotossensibilizador e a aplicação com um comprimento de onda correto e específico leva ao sucesso desta terapia (Habashneh et al., 2019). Devido à sua segurança, sem efeitos colaterais e mais aceitabilidade dos métodos não cirúrgicos de tratamento para os pacientes, a TFD é importante como um método terapêutico adjuvante com RAR, a fim de aumentar a eficiência dos tratamentos não cirúrgicos (Meimandi et al., 2017).

Figura 1 - Esquema da TFD.

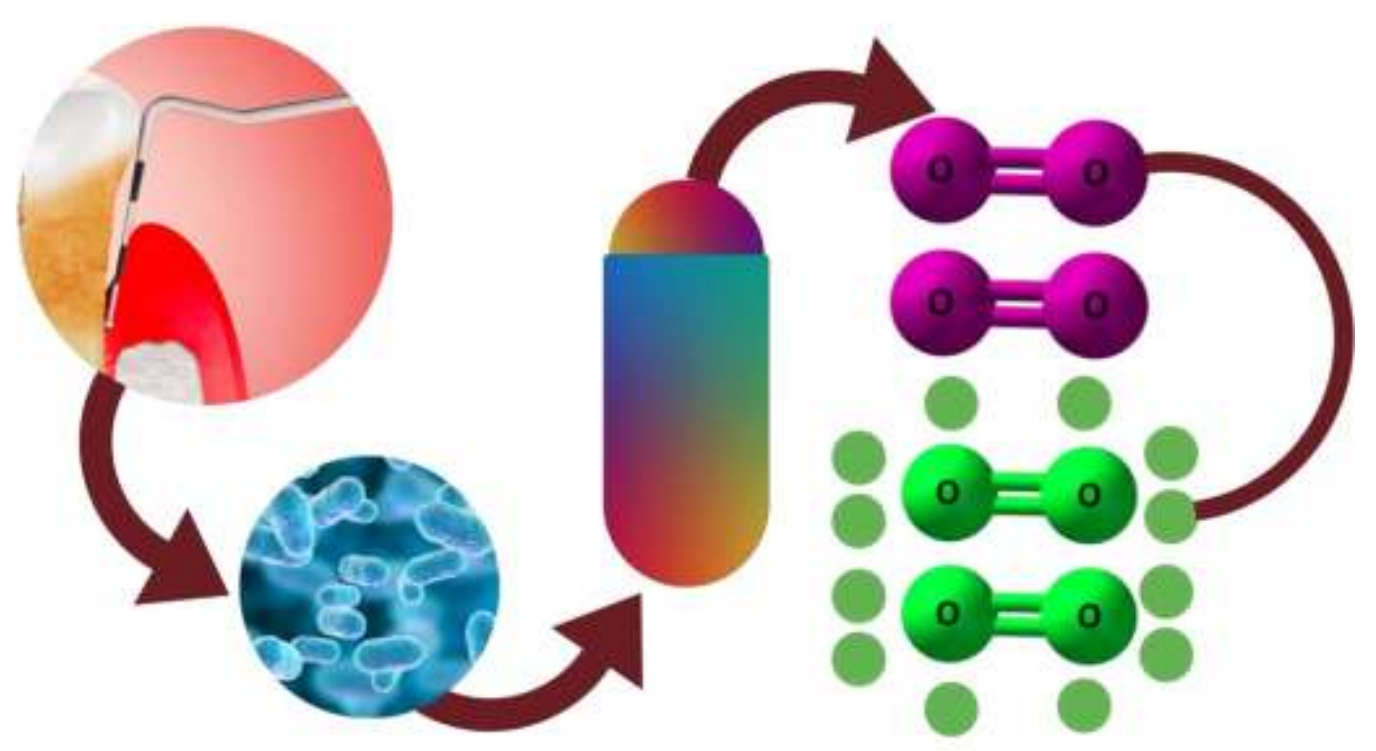

Fonte: Autores (2021).

No esquema acima apresentado é possível observar a presença de bactérias no elemento dental com doença periodontal. Ao ser adicionado um corante fotoativo (fotossensibilizador) ativado pela luz de um comprimento de onda específico, ocorre uma mudança de cadeias de $\mathrm{O}^{2}$ (na cor roxa) para espécies reativas citotóxicas de oxigênio como é representado pela cor verde.

\section{Resultados}

Foi realizado um levantamento bibliográfico das referências que estarão apresentadas na Tabela 1.A presente revisão de literatura, se deu através de uma coleta dos dados minuciosa com a utilização das palavras chaves descritas no presente trabalho. Seguindo os critérios de inclusão, foram identificados no Scielo um total de 108 referências bibliográficas, sendo selecionados 06 artigos. Já no Lilacs foram identificadas 382 referências sendo considerados apenas 02 artigos. No PubMed, foram identificadas 187 referências, permanecendo 30 artigos. A partir disso, foi realizada a leitura na integra, sendo selecionados 38 artigos para contemplar o presente estudo. 
Tabela 1. Resultados do levantamento bibliográfico.

\begin{tabular}{|c|c|c|}
\hline AUTOR/ANO & TÍTULO & RESULTADOS \\
\hline Castano et al., 2004 & $\begin{array}{l}\text { Mechanisms in photodynamic therapy: part one- } \\
\text { hotosensitizers, photochemistry and cellular } \\
\text { pcalization. }\end{array}$ & $\begin{array}{l}\text { Desenvolvidas novas fontes de luz mais fáceis de usar e, } \\
\text { frequentemente são baseadas em diodos de laser de } \\
\text { estado sólido, bem como diodos emissores de luz } \\
\text { baratos e lâmpadas de banda larga filtradas. }\end{array}$ \\
\hline Giannelli et al., 2019 & $\begin{array}{l}\text { Terapia fotônica em doenças periodontais uma } \\
\text { visão geral com avaliação da literatura e } \\
\text { recomendações de tratamento fundamentadas. }\end{array}$ & $\begin{array}{l}\text { Ao realizarem um estudo profundo dos corantes, } \\
\text { observaram que algumas bactérias, especialmente as } \\
\text { Gram-negativas, podem induzir a dimerização de } \\
\text { corantes fenotiazínicos, aumentando assim a absorção } \\
\text { de luz, as reações fotoquímicas e os efeitos bactericidas } \\
\text { gerais. }\end{array}$ \\
\hline $\begin{array}{l}\text { Mielczarek-Badora e } \\
\quad \text { Szulc, } 2013\end{array}$ & $\begin{array}{l}\text { Photodynamic therapy and its role in periodontitis } \\
\text { treatment. }\end{array}$ & $\begin{array}{l}\text { Observou que não elimina por completo os patógenos } \\
\text { periodontais, especialmente em bolsas periodontais } \\
\text { profundas. }\end{array}$ \\
\hline $\begin{array}{l}\text { Ramanauskaite } \text { et al., } \\
2020\end{array}$ & $\begin{array}{l}\text { The efficacy of adjunctive aids in periodontal } \\
\text { maintenance therapy: a systematic literature review } \\
\text { and meta-analysis. }\end{array}$ & $\begin{array}{l}\text { Constatou que a associação da RAR com a TFD gera } \\
\text { resultados na redução da profundidade de sondagem } \\
\text { com ganho significativo na inserção clínica. }\end{array}$ \\
\hline CAO et al., 2019 & $\begin{array}{l}\text { Efeito da terapia periodontal não cirúrgica no } \\
\text { controle glicêmico do diabetes mellitus tipo 2: uma } \\
\text { revisão sistemática e meta-análise da rede } \\
\text { bayesiana. }\end{array}$ & $\begin{array}{l}\text { Pacientes com periodontite crônica (não fumantes) tem } \\
\text { melhores resultados na utilização desta terapia, } \\
\text { alterando a glicemia nos que apresentam diabetes } \\
\text { melítus tipo } 2\end{array}$ \\
\hline Wang et al., 2009 & $\begin{array}{l}\text { Adjunctive photodynamic therapy improves the } \\
\text { outcomes of peri-implantitis: a randomized } \\
\text { controlled trial. }\end{array}$ & $\begin{array}{l}\text { Em pacientes com peri-implantite foi observado que, } \\
\text { após um dimensionamento da superfície onde é } \\
\text { aplicada, ocorre melhores resultados com redução de } \\
\text { forma significativa no índice de placa, sangramento } \\
\text { sulcular e perda de inserção clínica, ressaltando a } \\
\text { importância de estudar os efeitos dos corantes em } \\
\text { infecções peri-implantares. }\end{array}$ \\
\hline Wadhwa et al.,2021 & $\begin{array}{l}\text { Novel indocyanine green mediated antimicrobial } \\
\text { photodynamic therapy in the management of } \\
\text { chronic periodontitis - a randomized controlled } \\
\text { clinico-microbiological pilot study. }\end{array}$ & $\begin{array}{l}\text { As perspectivas futuras para TFD são inúmeras, visto } \\
\text { que técnicas e estudos têm surgido sobre este advento } \\
\text { da odontologia moderna. }\end{array}$ \\
\hline
\end{tabular}

Fonte: Autores (2021).

\section{Discussão}

De acordo com Giannelli et al. (2019) os primeiros instrumentos desenvolvidos para utilização na TFD foram os lasers, com propriedades únicas de emissão de um feixe de luz coerente e colimador com comprimento de onda sincronizado monocromático. De modo conjunto, Castano et al. (2004) relatam que tem sido desenvolvidas novas fontes de luz mais fáceis de usar e, frequentemente são baseadas em diodos de laser de estado sólido, bem como diodos emissores de luz baratos e lâmpadas de banda larga filtradas.

Segundo Mallineni et al. (2020) a TFD é uma alternativa para ser utilizada em médio e longo prazo. Giannelli et al. (2019), ao realizarem um estudo profundo dos corantes, observaram que algumas bactérias, especialmente as Gram-negativas, podem induzir a dimerização de corantes fenotiazínicos, aumentando assim a absorção de luz, as reações fotoquímicas e os efeitos bactericidas gerais.

Oliveira et al. (2017) apontam que a eficácia da TFD ocorre em bactérias Gram-positivas e Gram-negativas, evidenciando o fato que quanto menor a complexidade da membrana externa, maior será a facilidade de destruição celular. Entretanto, Mielczarek-Badora e Szulc (2013) afirmam que não elimina por completo os patógenos periodontais, especialmente em bolsas periodontais profundas.

A partir dos dados investigados, Ramanauskaite et al. (2020) observam que foi possível constatar que a associação da RAR com a TFD gera resultados na redução da profundidade de sondagem com ganho significativo na inserção clínica. Da 
mesma forma, Romeu et al. (2016) relatam que essa terapia conjunta apresenta maior e mais rápida cicatrização, sendo considerado um método confiável.

Estudos mais recentes afirmam que pacientes com periodontite crônica (não fumantes) tem melhores resultados na utilização desta terapia, alterando a glicemia nos que apresentam diabetes melítus tipo 2 (CAO et al., 2019). Para Macedo et al. (2014), nesses pacientes, faz-se necessário a realização de mais de uma aplicação para observar uma eficiente redução da hemoglobina glicada.

De acordo com Wang et al. (2009), em pacientes com peri-implantite foi observado que, após um dimensionamento da superfície onde é aplicada, ocorre melhores resultados com redução de forma significativa no índice de placa, sangramento sulcular e perda de inserção clínica, ressaltando a importância de estudar os efeitos dos corantes em infecções peri-implantares. Para Wadhwa et al. (2021) as perspectivas futuras para TFD são inúmeras, visto que técnicas e estudos têm surgido sobre este advento da odontologia moderna.

\section{Considerações Finais}

Embora evidências demonstrem a plena eficácia da Terapia Fotodinâmica, ainda é necessário a realização de estudos mais voltados para a sua utilização no tratamento de doenças periodontais como um todo. Sendo imprescindível a definição de protocolos terapêuticos específicos que permitam uma execução baseada na ciência. Dessa forma, o presente artigo traz de forma abrangente a aplicação deste importante método terapêutico que atua de forma auxiliar para o desbridamento e alisamento radicular. Apresentando assim, um resultado significativo e satisfatório no tratamento das doenças do periodonto.

\section{Agradecimentos}

Agradecemos a todos que direta ou indiretamente contribuíram para realização e sucesso do artigo.

\section{Referências}

Al-Hamoudi N. (2017). Is antimicrobial photodynamic therapy an effective treatment for chronic periodontitis in diabetes mellitus and cigarette smokers: a systematic review and meta-analysis. Photodiagnosis and photodynamic therapy, 19, 375-382. https://doi.org/10.1016/j.pdpdt.2017.05.018.

Allison, R. R., Bagnato, V. S., Cuenca, R., Downie, G. H., \& Sibata, C. H. (2006). The future of photodynamic therapy in oncology. Future oncology (London, England), 2(1), 53-71. https://doi.org/10.2217/14796694.2.1.53.

Cao, R., Li, Q., Wu, Q., Yao, M., Chen, Y., \& Zhou, H. (2019). Efeito da terapia periodontal não cirúrgica no controle glicêmico do diabetes mellitus tipo 2: uma revisão sistemática e meta-análise da rede Bayesiana. BMC oral health, 19 (1), 176. https://doi.org/10.1186/s12903-019-0829-y.

Carrera, E. T., Dias, H. B., Corbi, S., Marcantonio, R., Bernardi, A., Bagnato, V. S., Hamblin, M. R., \& Rastelli, A. (2016). The application of antimicrobial photodynamic therapy (aPDT) in dentistry: a critical review. Laser physics, 26(12), 123001. https://doi.org/10.1088/1054-660X/26/12/123001.

Castano, A. P., Demidova, T. N., \& Hamblin, M. R. (2004). Mechanisms in photodynamic therapy: part one-photosensitizers, photochemistry and cellular localization. Photodiagnosis and photodynamic therapy, 1(4), 279-293. https://doi.org/10.1016/S1572-1000(05)00007-4.

Cobb, C. M. (2006). Lasers in periodontics: a review of the literature. Journal of periodontology, 77(4), 545-564.

Estrela, C. (2018). Metodologia Científica: Ciência, Ensino, Pesquisa. Editora Artes Médicas.

Fernandes, L. A., de Almeida, J. M., Theodoro, L. H., Bosco, A. F., Nagata, M. J., Martins, T. M., Okamoto, T., \& Garcia, V. G. (2009). Treatment of experimental periodontal disease by photodynamic therapy in immunosuppressed rats. Journal of clinical periodontology, 36(3), 219-228. https://doi.org/10.1111/j.1600-051X.2008.01355.x.

Garg AD, Maes H, Romano E, \& Agostinis P. Autophagy a major adaptation pathway shaping cancer cell death and anti cancer immunity responses following photodynamic therapy. Photochem Photobiol Sci. 14:1410-1424. https://doi.org/10.1039/C4PP00466C.

Giannelli, M., Lasagni, M., \& Bani, D. (2019). Terapia fotônica em doenças periodontais uma visão geral com avaliação da literatura e recomendações de tratamento fundamentadas. Jornal internacional de ciências moleculares, 20 (19), 4741. https://doi.org/10.3390/ijms20194741.

Gillies, M., Ranakusuma, A., Hoffmann, T., Thorning, S., McGuire, T., Glasziou, P., \& Del Mar, C. (2015). Common harms from amoxicillin: a systematic review and meta-analysis of randomized placebo-controlled trials for any indication. CMAJ : Canadian Medical Association journal = journal de l'Association medicale canadienne, 187(1), E21-E31. https://doi.org/10.1503/cmaj.140848. 
Habashneh, RA, Mashal, MA, Khader, Y., \& Qudah, R. (2019). Efeitos clínicos e biológicos da terapia fotodinâmica adjuvante na periodontite refratária. Journal of lasers in medical sciences, 10 (2), 139-145. https://doi.org/10.15171/jlms.2019.22.

Javali, M. A., AlQahtani, N. A., Ahmad, I., \& Ahmad, I. (2019). Antimicrobial photodynamic therapy (light source; methylene blue; titanium dioxide): Bactericidal effects analysis on oral plaque bacteria: An in vitro study. Nigerian journal of clinical practice,22(12), 1654-1661. https://doi.org/10.4103/njcp.njcp_189_19.

Jiang, C., Yang, W., Wang, C., Qin, W., Ming, J., Zhang, M., Qian, H., \& Jiao, T. (2019). Methylene Blue-Mediated Photodynamic Therapy Induces Macrophage Apoptosis via ROS and Reduces Bone Resorption in Periodontitis. Oxidative medicine and cellular longevity, $2019,1529520$. https://doi.org/10.1155/2019/1529520.

Konopka, K., \& Goslinski, T. (2007). Photodynamic therapy in dentistry. Journal of dental research,86(8), 694-707. https://doi.org/10.1177/154405910708600803.

Kumar, V., Sinha, J., Verma, N., Nayan, K., Saimbi, C. S., \& Tripathi, A. K. (2015). Scope of photodynamic therapy in periodontics. Indian journal of dental research : official publication of Indian Society for Dental Research, 26(4), 439-442. https://doi.org/10.4103/0970-9290.167636.

Macedo, G., Novaes, A. B., Jr, Souza, S. L., Taba, M., Jr, Palioto, D. B., \& Grisi, M. F. (2014). Additional effects of aPDT on nonsurgical periodontal treatment with doxycycline in type II diabetes: a randomized, controlled clinical trial. Lasers in medical science, 29(3), 881-886. https://doi.org/10.1007/s10103-013-1285-6.

Mallineni, S., Nagarakanti, S., Gunupati, S., Bv, RR, Shaik, MV, \& Chava, VK (2020). Efeitos clínicos e microbiológicos da terapia adjuvante com laser de diodo fotodinâmico no tratamento da periodontite crônica: um ensaio clínico randomizado. Journal of Dental Research, Dental Clinics, Dental Prossers , 14 (3), 191-197. https://doi.org/10.34172/joddd.2020.030

Meimandi, M., Talebi Ardakani, M. R., Esmaeil Nejad, A., Yousefnejad, P., Saebi, K., \& Tayeed, M. H. (2017). The Effect of Photodynamic Therapy in the Treatment of Chronic Periodontitis: A Review of Literature. Journal of lasers in medical sciences, 8(Suppl 1), S7-S11. https://doi.org/10.15171/jlms.2017.s2.

Meisel, P., \& Kocher, T. (2005). Terapia fotodinâmica para doenças periodontais: estado da arte. Journal of photochemistry and photobiology B: Biology, 79 (2), 159-170.

Mielczarek-Badora, E., \& Szulc, M. (2013). Photodynamic therapy and its role in periodontitis treatment. Postepy higieny i medycyny doswiadczalnej (Online), 67, 1058-1065. https://doi.org/10.5604/17322693.1075915.

Moan, J., \& Berg, K. (1991). The photodegradation of porphyrins in cells can be used to estimate the lifetime of singlet oxygen. Photochemistry and photobiology, 53(4), 549-553. https://doi.org/10.1111/j.1751-1097.1991.tb03669.x.

Nagahara, A., Mitani, A., Fukuda, M., Yamamoto, H., Tahara, K., Morita, I., Ting, C. C., Watanabe, T., Fujimura, T., Osawa, K., Sato, S., Takahashi, S., Iwamura, Y., Kuroyanagi, T., Kawashima, Y., \& Noguchi, T. (2013). Antimicrobial photodynamic therapy using a diode laser with a potential new photosensitizer, indocyanine green-loaded nanospheres, may be effective for the clearance of Porphyromonas gingivalis. Journal of periodontal research, 48(5), 591-599. https://doi.org/10.1111/jre.12042.

Oliveira, C. L. de, Santos, K. de S., Fernandes Neto, J. de A., Batista, A. L. A., Medeiros, C. L. S. G. de, \& Catão, M. H. C. de V. (2017). A eficácia da terapia fotodinâmica no tratamento periodontal não cirúrgico. ARCHIVES OF HEALTH INVESTIGATION, 6(6). https://doi.org/10.21270/archi.v6i6.2072.

Park, D., Choi, E. J., Weon, K. Y., Lee, W., Lee, S. H., Choi, J. S., Park, G. H., Lee, B., Byun, M. R., Baek, K., \& Choi, J. W. (2019). Non-Invasive Photodynamic Therapy against -Periodontitis-causing Bacteria. Scientific reports, 9(1), 8248. https://doi.org/10.1038/s41598-019-44498-4.

Petersilka, G. J., Tunkel, J., Barakos, K., Heinecke, A., Häberlein, I., \& Flemmig, T. F. (2003). Subgingival plaque removal at interdental sites using a lowabrasive air polishing powder. Journal of periodontology, 74(3), 307-311. https://doi.org/10.1902/jop.2003.74.3.307.

Prażmo, E. J., Kwaśny, M., Łapiński, M., \& Mielczarek, A. (2016). Photodynamic Therapy As a Promising Method Used in the Treatment of Oral Diseases. Advances in clinical and experimental medicine: official organ Wroclaw Medical University, 25(4), 799-807. https://doi.org/10.17219/acem/32488.

Qi, M., Chi, M., Sun, X., Xie, X., Weir, MD, Oates, TW, Zhou, Y., Wang, L., Bai, Y., \& Xu, HH (2019). Novas terapias fotodinâmicas antibacterianas baseadas em nanomateriais para combater biofilmes bacterianos orais e doenças infecciosas. International journal of nanomedicine, 14, 69376956. https://doi.org/10.2147/IJN.S212807.

Raghavendra, M., Koregol, A., \& Bhola, S. (2009). Photodynamic therapy: a targeted therapy in periodontics. Australian dental journal, 54 Suppl 1, S102S109. https://doi.org/10.1111/j.1834-7819.2009.01148.x.

Rajesh, S., Koshi, E., Philip, K., \& Mohan, A. (2011). Antimicrobial photodynamic therapy: An overview. Journal of Indian Society of Periodontology, 15(4), 323-327. https://doi.org/10.4103/0972-124X.92563.

Ramanauskaite, E., Sakalauskaite, U. M., \& Machiulskiene, V. (2020). The Efficacy of Adjunctive Aids in Periodontal Maintenance Therapy: A Systematic Literature Review and Meta-analysis. Oral health \& preventive dentistry, 18(1), 889-910. https://doi.org/10.3290/j.ohpd.a45406.

Romeo, U., Nardi, G. M., Libotte, F., Sabatini, S., Palaia, G., \& Grassi, F. R. (2016). The Antimicrobial Photodynamic Therapy in the Treatment of PeriImplantitis. International journal of dentistry, 2016, 7692387. https://doi.org/10.1155/2016/7692387.

Sgolastra, F., Petrucci, A., Gatto, R., Marzo, G., \& Monaco, A. (2013). Photodynamic therapy in the treatment of chronic periodontitis: a systematic review and meta-analysis. Lasers in medical science, 28(2), 669-682. https://doi.org/10.1007/s10103-011-1002-2.

Tanner AC. Anaerobic culture to detect periodontal and caries pathogens. J Oral Biosci. 2015;57(1):18-26. 10.1016/j.job.2014.08.001. 
Research, Society and Development, v. 10, n. 15, e277101522931, 2021

(CC BY 4.0) | ISSN 2525-3409 | DOI: http://dx.doi.org/10.33448/rsd-v10i15.22931

Tsai, C. Y., Tang, C. Y., Tan, T. S., Chen, K. H., Liao, K. H., \& Liou, M. L. (2018). Subgingival microbiota in individuals with severe chronic periodontitis. Journal of microbiology, immunology, and infection = Wei mian yu gan ran za zhi, 51(2), 226-234. https://doi.org/10.1016/j.jmii.2016.04.007.

Wadhwa, A., Mallapragada, S., \& Sharma, P. (2021). Novel indocyanine green mediated antimicrobial photodynamic therapy in the management of chronic periodontitis - A randomized controlled clinico-microbiological pilot study. Journal of oral biology and craniofacial research, 11(1), 57-62. https://doi.org/10.1016/j.jobcr.2020.11.005.

Wang, H., Li, W., Zhang, D., Li, W., \& Wang, Z. (2019). Adjunctive photodynamic therapy improves the outcomes of peri-implantitis: a randomized controlled trial. Australian dental journal, 64(3), 256-262. https://doi.org/10.1111/adj.12705.

Zhao, Y., Pu, R., Qian, Y., Shi, J., \& Si, M. (2021). Antimicrobial photodynamic therapy versus antibiotics as an adjunct in the treatment of periodontitis and peri-implantitis: A systematic review and meta-analysis. Photodiagnosis and photodynamic therapy, 34, 102231. https://doi.org/10.1016/j.pdpdt.2021.102231. 